\title{
Umstellen oder Augmentieren bei Nichtansprechen auf Antidepressiva?
}

Fragestellung: Wie wirksam ist bei Nichtansprechen auf ein Antidepressivum eine Umstellung auf Bupropion, eine zusätzliche Gabe von Bupropion oder eine zusätzliche AripiprazolGabe?

Hintergrund: Weniger als ein Drittel der depressiven Patienten erreichen unter dem ersten Antidepressivum eine Remission ihrer Symptomatik. Daher sind Studien begrüßenswert, die Switch- oder Augmentierungsstrategien bei Nonresponse auf Antidepressiva untersuchen.

Patienten und Methodik: In die Studie wurden 1.522 Patienten mit nicht psychotischer Depression, die auf mindestens ein Antidepressivum nicht angesprochen hatten, auf einen der drei Behandlungsarme randomisiert: 1) Umstellung auf Bu-

Mohamed S, Johnson GR, Chen $\mathrm{P}$ et al. Effect of antidepressant switching vs augmentation on remission among patients with major depressive disorder unresponsive to antidepressant treatment. JAMA 2017; 318: $132-45$ propion ( $\mathrm{n}=511)$, 2) Augmentierung der gegenwärtigen antidepressiven Therapie mit Bupropion $(n=506)$ oder 3) Augmentierung mit Aripiprazol $(\mathrm{n}=505)$. Die Akutbehandlungsphase dauerte zwölf Wochen, anschließend wurden die Patienten für bis zu 36 Wochen nachbeobachtet. Der primäre Outcome war das Erreichen einer Remission, sekundäre Outcomes die Responseraten, Rückfälle und Nebenwirkungen.

Ergebnisse: Von den randomisierten Patienten waren $85 \%$ Männer, zirka 75 \% schlossen die Akutbehandlungsphase komplett ab. Die Remissionsraten nach zwölf Wochen waren in der Bupropion-Switchgruppe 22,3\%, in der Bupropion-Augmentierungsgruppe $26,9 \%$ und in der Aripiprazol-Augmentierungsgruppe 28,9\%. Die Remissionsrate war in der Aripiprazol-Gruppe der Switchgruppe knapp signifikant überlegen. Andere Vergleiche bezüglich der Remissionsrate waren nicht signifikant verschieden, ebenso nicht die Häufigkeit der Wiedererkrankungen. In der Aripiprazol-Augmentierung kam es häufiger zu Nebenwirkungen wie Müdigkeit, Akathisie und Gewichtszunahme.

Schlussfolgerungen: Die Augmentierung einer AntidepressivaTherapie mit Aripiprazol führt zu einer statistisch signifikanten, jedoch nur mäßigen Überlegenheit gegenüber einem Umstellen auf Bupropion. Aufgrund der kleinen Effektstärken und der Nebenwirkungen muss die Relevanz einer Augmentierung mit Aripiprazol weiter überprüft werden.

\section{- Kommentar von Klaus Lieb, Mainz}

\section{Leitlinien bestätigt}

Die vorliegende Studie ist eine Nachfolgestudie der berühmten STAR*D-Studie in den USA, in der sich Bupropion als mindestens so effektiv wie andere Switch- oder Augmentierungsstrategien erwiesen hatte, allerdings war damals die Studie nicht so angelegt, dass die Bupropion-Strategien statistisch ausreichend gepowert waren. Zusätzlich gibt es Hinweise, dass Aripiprazol als Augmentierungsstrategie bei Nonresponse auf Antidepressiva wirksam sein könnte [1, 2]. Die Autoren führten daher diese Studie durch, deren größte Schwäche darin liegt, dass sie keinen Studienarm mit fortgesetzter Antidepressivatherapie (mit Placebo) hatte. So weiß man nicht, ob ein Switch zu Bupropion der fortgesetzten Therapie überhaupt überlegen war. Insgesamt sind die durch die drei Strategien erreichten Remissionsraten zwischen $22 \%$ und $29 \%$ gering, mit einer leichten Überlegenheit für die Augmentierung mit Aripiprazol. Klinisch wird die S3-Leitlinie für Depression bestätigt, die das Umstellen auf ein anderes Antidepressivum nicht als Mittel der Wahl bei Nonresponse auf ein erstes Antidepressivum empfiehlt. Zu bevorzugen ist eine Augmentierung mit Mirtazapin oder mit Lithium. Von der Kombination mit Bupropion hätte ich mir persönlich in dieser Studie mehr erwartet, da klinisch oft argumentiert wird, dass insbesondere SSRI von der Kombination mit einem Noradrenalin- und Dopamin-Wiederaufnahmehemmer profitieren sollten.

Fazit: Eine Augmentierung mit Aripiprazol ist etwas besser, als auf Bupropion umzustellen. Ob das die vermehrten Nebenwirkungen rechtfertigt, muss weiter untersucht werden.
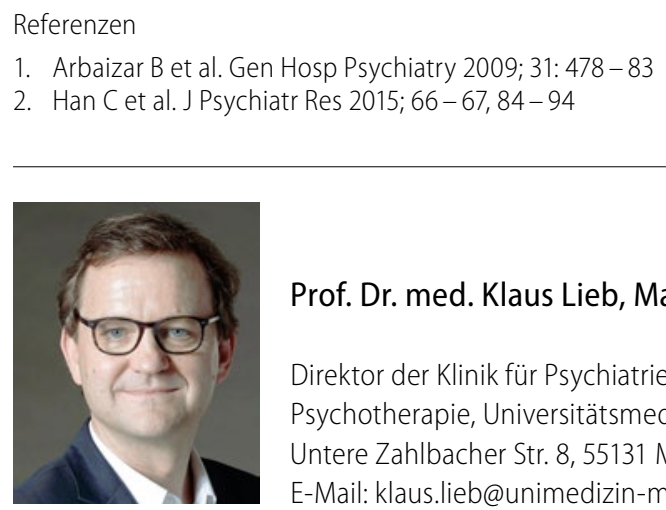

Prof. Dr. med. Klaus Lieb, Mainz

Direktor der Klinik für Psychiatrie und Psychotherapie, Universitätsmedizin Mainz Untere Zahlbacher Str. 8, 55131 Mainz E-Mail: klaus.lieb@unimedizin-mainz.de 